\title{
Editor's note: Special Issue on Mobile Ad-hoc and Sensor Networks
}

Peer-to-Peer Networking and Applications gratefully acknowledges the editorial work of the scholars listed below on the special issue entitled "Mobile Ad-hoc and Sensor Networks":

Weili (Lily) Wu, University of Texas at Dallas, USA

Jie Li, University of Tsukuba, Japan

The 8 papers in this issue include:

"Events detection and community partition based on probabilistic snapshot for evolutionary social network" by Zhongnan Zhang, Lei Hu, Ming Qiu and Fangyuan Gao

"Fault diagnosis of body sensor networks using hidden Markov model" by Haibin Zhang, Jiajia Liu, Rong Li, and Hua Le

"Connected sensor cover and related problems" by Yingfan L. Du and Lidong Wu
"Two-layer hybrid peer-to-peer networks" by Zhenhua Duan, Cong Tian, Mengchu Zhou, Xiaobing Wang, Nan Zhang, Hongwei Du and Lei Wang

"Using multi-features to recommend friends on locationbased social networks" by Gao Xu-Rui, Wang Li and Wu Wei-Li

"A novel broadcast authentication protocol for internet of vehicles" by Na Ruan, Mengyuan Li and Jie Li

"Analysis to reveal evolution and topological features of a real mobile social network" by Qichao Xu, Zhou Su, Zejun Xu, Dongfeng Fang and Bo Han

Interference-constrained routing over P2P-share enabled multi-hop D2D networks" by Qinghe Du, Meng Liu, Qian Xu, Houbing Song, Li Sun and Pinyi Ren 